\title{
CONF-921101--141
}

\section{A TEM STUDY OF STEP MORPHOLOGY ON THE NON-UHV HEAT-TREATED $\mathrm{MgO}$ (100) SURFACE}

\author{
SIMON KING AND C. BARRY CARTER
}

Dept. of Chemical Engineering and Materials Science, University of Minnesota, Amundson Hall, 421 Washington Ave. S.E., Minneapolis, MN 55455

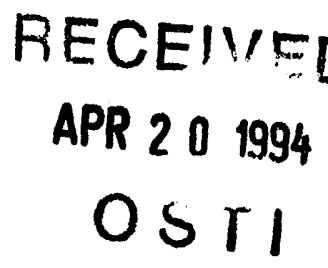

\section{ABSTRACT}

Preliminary results from a study of the morphology of the $\mathrm{MgO}$ (100) surface after chemical etching followed by heat-treatment in air are presented. Contrast arising from the surface-steps so formed is found to be strongly dependent on the deviation parameter, suggesting that many of the step-faces are not perpendicular to the $(100)$ foil plane but are, instead, often rather shallowly inclined.

\section{INTRODUCTION}

The (100) surface of $\mathrm{MgO}$, which is easily formed by cleaving owing to its low surface energy, is now in widespread use as a substrate for the deposition particularly of high- $T_{C}$ superconductors (e.g., [1,2]), as well as of other ceramics such as BN [3]. The importance of the topography of the substrate surface is well documented: Examples include the observation of the nucleation and "island-growth" mechanism of laser-ablated $\mathrm{YBa}_{2} \mathrm{Cu}_{3} \mathrm{O}_{7-\mathrm{x}}(\mathrm{YBCO})[1,4]$, and an improvement in superconducting properties for $\mathrm{YBCO}$ thin-films deposited onto annealed as opposed to unannealed $\mathrm{MgO}$ substrates [5]. Surface irregularities on (100) $\mathrm{MgO}$ surfaces also may play key roles in many reactions for which $\mathrm{MgO}$ is an efficient catalyst (e.g., [6,7]).

Surface steps formed during cleavage, commonly of atomic height, have been observed in Reflection Electron Microscopy (REM) investigations [8] to be accurately aligned along $\langle 100\rangle$ directions. For $\mathrm{MgO}$ smoke-particle platelets, two different step morphologies have been identified by TEM, namely, atomic height steps which are curved, and hence lie along no particular direction, and those a few unit cells in height which apparently enclose rectangular terraces [9]. Straight steps $\sim 2$ unit cells high, with edges along $<100>$ have also been imaged by High-Resolution ElectronMicroscopy (HREM) at the peripheries of $\mathrm{MgO}$ smoke microcubes [10]. In the preparation of selfsupporting foils for deposition, however, chemical washing followed by annealing has been shown to produce a high density of 'large' steps. These steps may be straight or curved and do not, apparently, lie along any particular crystallographic orientation $[1,2,4,11]$. An understanding of the morphology of these steps is, therefore, fundamental to the characterization of these $\mathrm{MgO}$ foils.

\section{EXPERMMNTAL}

Thin TEM foils have been prepared by the method of Norton et al [4,11]. Briefly, an $\mathrm{MgO}$ single crystal is cleaved parallel to $(100)$, ground to a thickness $\sim 100 \mu$, dimpled to $20 \mu$ prior to ionmilling, with 4kV Ar ions in a turbo-pumped Gatan ion-mill. Milling damage is removed by a succession of acid treatments prior to heat-treatment at $1350^{\circ} \mathrm{C}$ in air. TEM is performed in a Philips $\mathrm{CM} 30$, operated at $200 \mathrm{kV}$ to minimize sub-threshold radiation damage. Results from two specimens, annealed for 10 and 15.5 minutes, respectively, are shown here. These represent some of the key features of a larger study of the dependence of surface morphology on heat treatment.

\section{RESULTS}

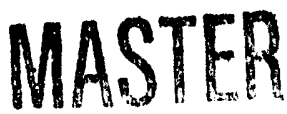

Some of the features common to these acid-treated and annealed $\mathrm{MgO}$ thin-foils are shown in fig. 1. Both images in this figure are bright-field (BF) micrographs taken with the incident beam exactly parallel to the [100] zone axis. The foils of figures $1(\mathrm{a})$ and $1(\mathrm{~b})$ have been heat-treated for 10 and 15.5 minutes at $1350^{\circ} \mathrm{C}$, respectively. The orientation depicted by the Thompson tetrahedron of figure 1(a) also applies to figure 1(b) and figure 2.

The general morphology of these foils consists of a high density of pores within the thin, electron-transparent regions. These pores result from the acid treatment necessary to remove the ionmilling damage. As can readilv be seen in both images $1(\mathrm{a})$ and $1(\mathrm{~b})$. the Dores are generallv faceted 


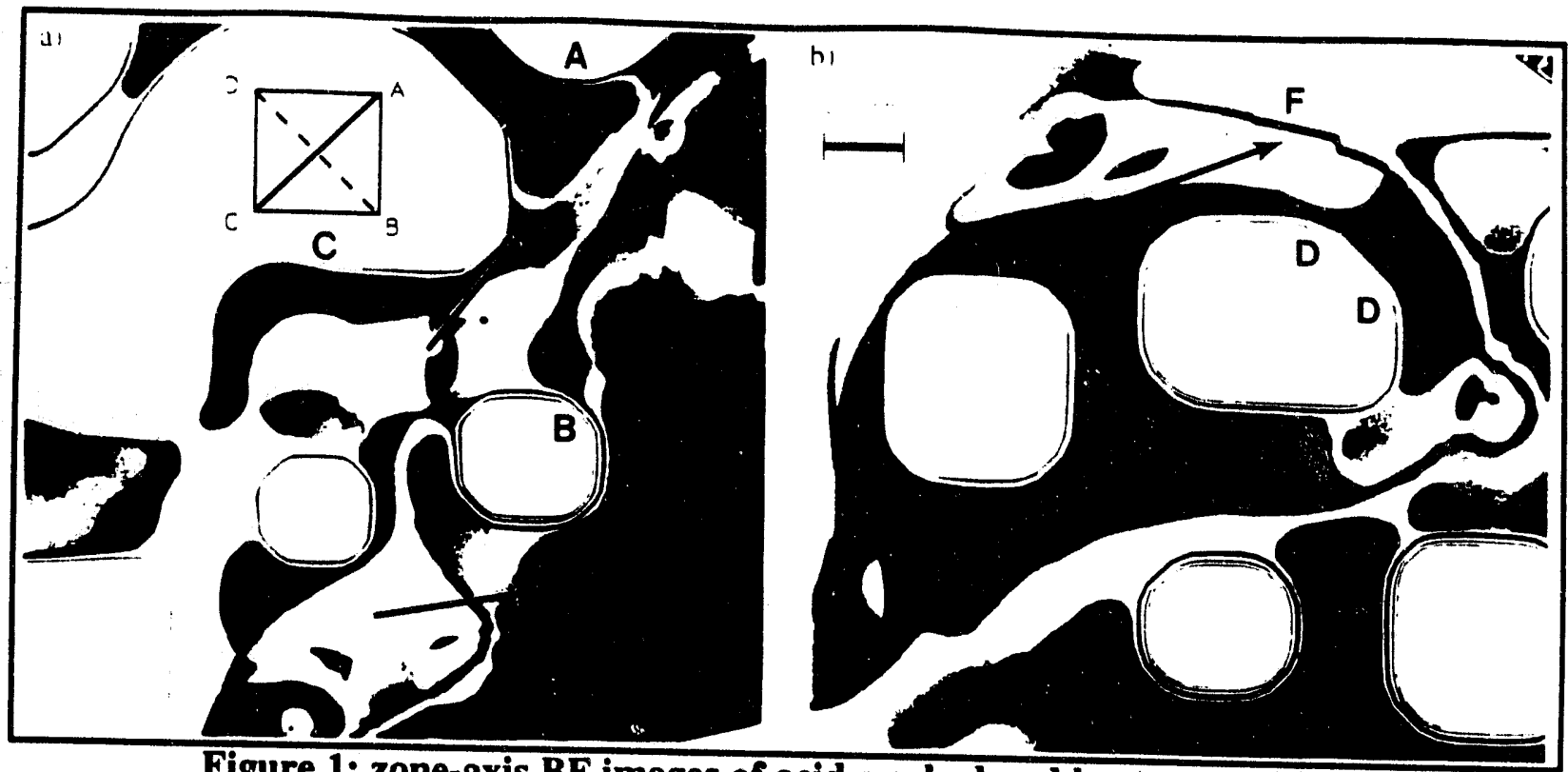

Figure 1: zone-axis BF images of acid-washed and heat-treated foils.

parallel to $<100\rangle$. Notice also that where the otherwise curved edge of the foil passes through the [100] orientation at ' $A$ ', a facet parallel to [100] is formed. Around the pores, facets parallel to $<110\rangle$ (e.g., [011] at ' $B$ ) also are found. Micro-facets, consisting of segments parallel to $\langle 100\rangle$ directions interspersed with segments lying along higher index directions such as at ' $C$ ' are common in regions of the edge, or of particularly large pores, with orientations within $\sim 10^{\circ}$ or so of a $\langle 100\rangle$ direction.

Higher index facets around some pores are also in evidence (e.g. at ' $D$ ).

The abrupt changes in the background contrast in these foils indicate the presence of surface steps. Regions of constant contrast, which generally represent terraces of constant thickness, may range up to hundreds of nanometers across. However, the inspection of almost any micrograph of these heat-treated (100)-oriented $\mathrm{MgO}$ foils (e.g., $[1,4,12])$ shows several narrow contrast bands which might, at first, be taken as arising from narrow terraces bounded by closely-spaced steps. Examples of these bands, arrowed in figure 1, range from $\sim 50 \AA$ (e.g.,' $E$ ) to $\sim 350 \AA$ (e.g., ' $F$ ) across. In these particular foils, the step traces are generally highly curved and, hence, are not aligned along any particular direction.

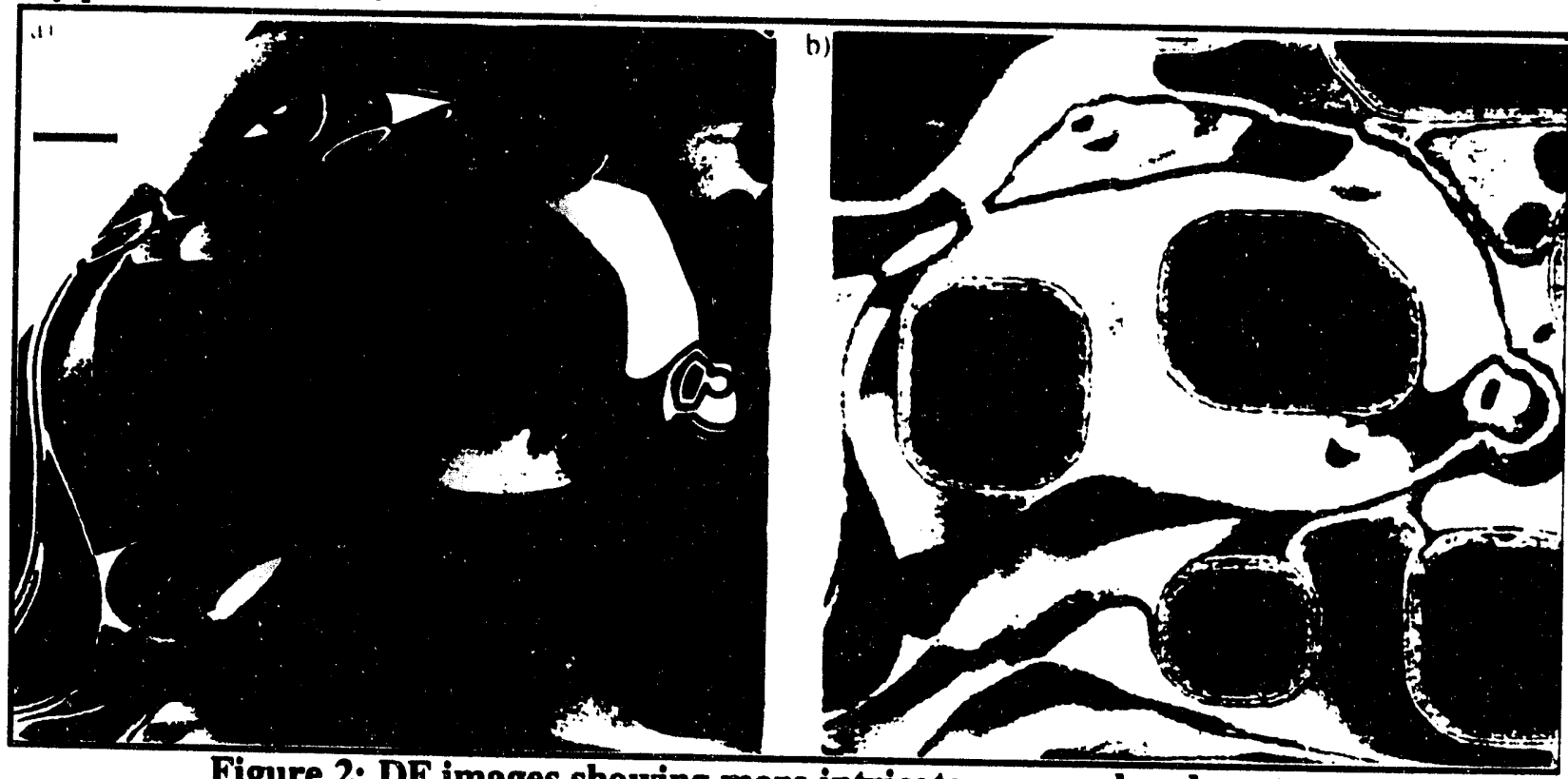

Figure 2: DF images showing more intricate narrow-band contrast.

The narrow contrast bands of figure 1(b) are investigated further in figure 2 , which consists of two dark-field (DF) images taken with an operating reflection of $\tilde{g}=[022]$ and at a foil orientation approximately $4^{\circ}$ away from [100] towards [211]. In figure 2(a), which has the larger deviation parameter of the two, several more narrow contrast bands are visible than was the case for figure 1(b) (some of the new bands are arrowed). Notice particularly that the large dark band (' $F$ ) 
now shows as a succession of six light and dark fringes. In figure 2(b), however, only 3 fringes are visible.

This region of the specimen is shown over a large $\left(-70^{\circ}\right)$ angle of tilt in figure 3 . The deviation parameters $(s)$ are: $1.58 \times 10^{-2} \AA^{-1}$ for micrographs (a) and (b) and $0.57 \times 10^{-2} \AA^{-1}$ for micrograph (c). The tilt axis, parallel to $\tilde{g}$, and foil orientations are represented schematically at the right of the figure. Tilting through this angle enables features on the top ('T) and bottom ('B') surfaces readily to be distinguished. It can be seen that the two similar large bands at the top of the figure are on different surfaces.

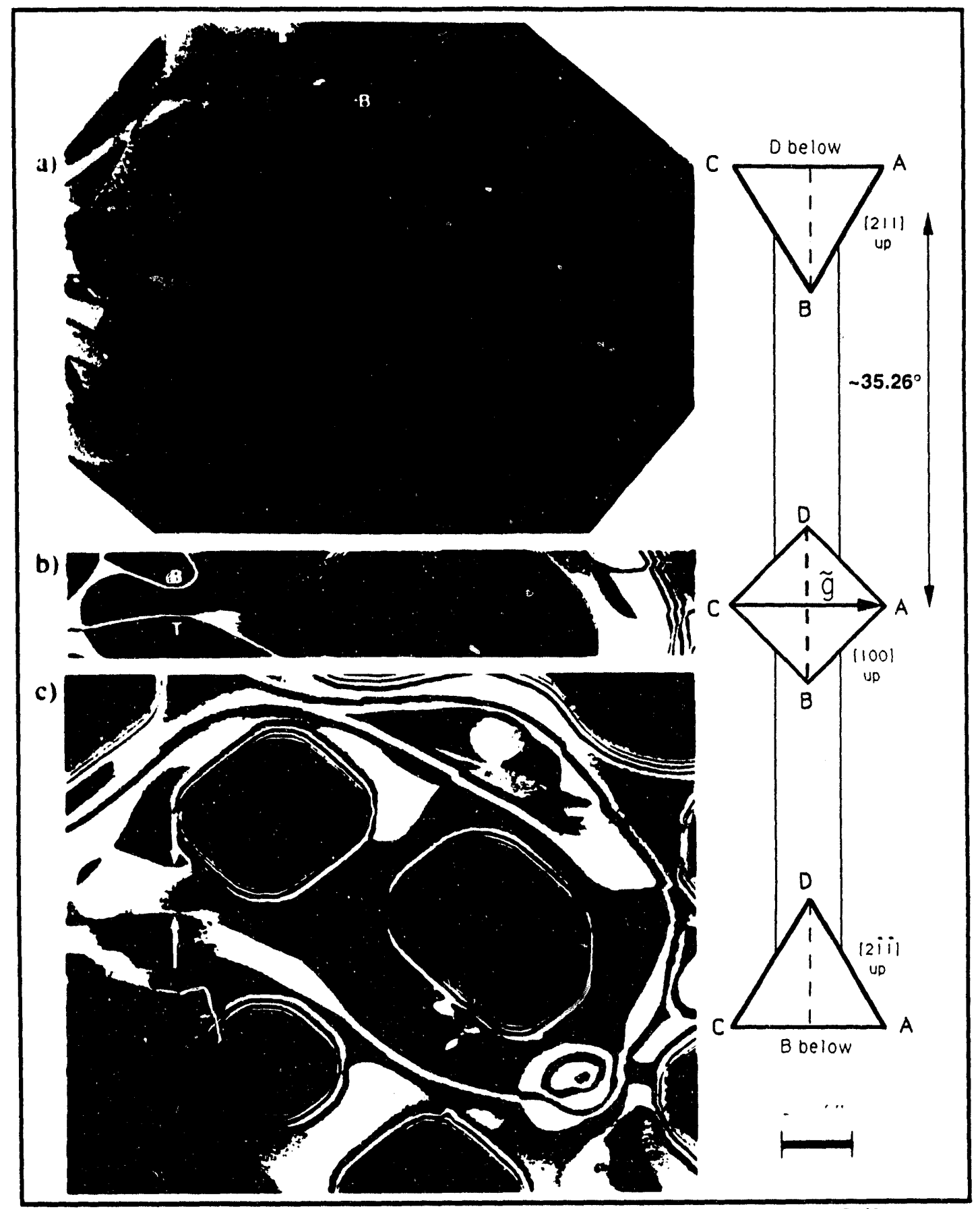

Figure 3: Near-weak-beam images taken over a large angle of tilt

While faceting of the step traces is generally not evident in these samples, two short $(\sim 550 \AA)$ segments (arrowed) are accurately aligned along [011] (their apparent direction does not alter as the foil is tilted about the [011] axis). These segments also lie on opposite surfaces and enable a rough estimate of the local foil thickness to be made $(\approx 900 \AA)$. Although this value is in general agreement with Convergent-Beam Electron-Diffraction (CBED) thickness measurements from an adjacent area [13], such estimates rely on the assumption that the observed contrast in any 
two images originates from exactly the same place, which, in this case, is thought to be unlikely. Notice, also, both that the width of this narrow contrast band pair is decreased where it passes through the $[011]$ orientation in figure $3(\mathrm{~b})$, and that this same band pair, which now appear dark, is extremely narrow in figure 3(a), in which the (111) plane is brought close to the vertical. This contrast behavior is consistent with the alignment of these features with, or close to, the (i11) plane.

\section{DISCUSSION}

Two possible explanations for the narrow contrast bands present themselves. The first is that they correspond directly to multiple, closely-spaced terraces. An alternative, and seemingiy more likely hypothesis is that these dark-light bands are simply extinction contours produced by thickness gradients. Were this to be the case, then as the effective extinction distance $\left(\xi_{\mathrm{B}}^{\mathrm{e}}\right)$ is reduced (e.g. by increasing $\boldsymbol{s}$ ) a greater number of more closely spaced fringes should become visible. This trend with increasing $S$ is clearly evident in figures $1(\mathrm{~b}), 2(\mathrm{~b})$ and 2(a), respectively. Briefly, other arguments supporting the latter conclusion are the absence of 'grey' narrow-bands (the shade of a band is always at a local extremum in $\left.\left|\Phi_{\mathrm{g}}\right|^{2}\right)$ and the lack of 'sharpness' from the edges of these bands and other step edges (even though other features such as pore edges or dislocations in weakbeam images can be seen with relative clarity). Given that these narrow bands arise from extinction fringes, it is an inescapable conclusion that step edges in these foils are inclined to the (100) plane.

In some cases, it is possible to estimate the angle made by an inclined face and the (100) surface of the thin foil from the separation of adjacent extinction contours contained wholly within a step face. For example, for the case of figure $2(a)$, for which $\xi_{\mathrm{g}}^{\mathrm{ef}} \approx 63 \AA$, and adjacent dark fringes at ' $F$ ' are separated by $\sim 204 \AA$, an angle of approximately $17^{\circ}$ is indicated. This angle appears to be relatively invariant $\left(\sim 14.5^{\circ}\right.$ to $\left.17.5^{\circ}\right)$ along the entire length of the two large inclined step-face traces $\left(' B^{\prime}, T^{\prime}\right.$ of figure 3(a)) which curve gradually through $\sim 270^{\circ}$. Hence, if faceting of these large step faces is occurring, which is not immediately apparent, very high-index planes would be involved.

Further evidence as to the inclination of the step faces to the (100) foil may be gleaned by noting that the projected widths of the step-faces (i.e., the contrast bands) are greater either side of those sections ' $B, T^{\prime}$ in figure $3(\mathrm{~b})$ which lie on, or close to, a $\{111\}$ plane. If sections $B, T^{\prime}$ are taken as representing the (111) plane, this increase in width constitutes direct evidence that neighboring parts of these step traces are inclined at substantially less than $54.7^{\circ}$ to the (100) foil surface. More accurate estimates of the inclination of these shorter steps to the (100) foil plane are in progress. However, the possibility is raised that many, most or perhaps even all of the steps seen in these chemically washed and heat-treated $\mathrm{MgO}$ foils are, in fact, rather shallowly inclined to (100).

\section{CONCLUSIONS}

1) Many of the steps in these acid washed and annealed in air MgO foils appear to have inclined faces. These inclined faces are recognizable by their characteristic "narrow-band" contrast.

2) The $<100\rangle$ faceting behavior exhibited by steps on cleaved $\mathrm{MgO}$, or on smoke particles has not been seen in these specimens.

3 ) The edges of pores and of the foil appear to facet readily along $<100\rangle$. There is also some early indication of both micro-faceting, and higher index faceting which remain to be fully characterized.

\section{REFERENCES}

[1] M.G.Norton, C.B.Carter; Scanning Microsc., 6, 385-398 (1992)

[2] M.G.Norton, C.B.Carter; J. Mater. Res., 5, 2762-2765 (1990)

[3] L.F.Allard, A.K.Datye, T.A.Nolan, S.L.Mahan, R.T.Paine; Ultramicrosc., 37, 153-168 (1991)

[4] M.G.Norton C.B.Carter; J. Cryst. Grov th 110, 641-651 (1991)

[5] B.H.Moeckly et al; Appl. Phys. Lett., 57, 1687-1689 (1990)

[6] H.Onishi, C.Egawa, T.Aruga, Y.Iwasawa; Surface Science, 191, 479-491 (1987)

[7] S.Ferrer, J.M.Rojo, M.Salmeron, G.A.Somorjai; Phil.Mag. A, 45 , 261-269 (1982)

[8] Z.L.Wang, J.Bentley, E.A.Kenik, L.L.Horton, R.A.McKee; Surface Science, 273, 88-108 (1992)

[9] G.Lehmpfuhl, Y.Uchida; Ultramicroscopy, 4, 275-282 (1979)

[10] T.Tanji, H.Masaoka, J.Ito, K.Yada, J.M.Cowley; Ultramicroscopy 27, 223-232 (1989)

[11] M.G.Norton, S.R.Summerfelt, C.B.Carter; Appl. Phys. Lett., 56, 2246-2248 (1990)

[12] I.M.Anderson - unpublished work.

[13] S.L.King, S.McKernan, C.B.Carter - these proceedings.

[14] The authors acknowledge the support of the D.O.E. under grant \# DE-FG02-89ER45381 


\section{DISCLAIMER}

This report was prepared as an account of work sponsored by an agency of the United States Government. Neither the United States Government nor any agency thereof, nor any of their employees, makes any warranty, express or implied, or assumes any legal liability or responsibility for the accuracy, completeness, or usefulness of any information, apparatus, product, or process disclosed, or represents that its use would not infringe privately owned rights. Reference herein to any specific commercial product, process, or service by trade name, trademark, manufacturer, or otherwise does not necessarily constitute or imply its endorsement, recommendation, or favoring by the United States Government or any agency thereof. The views and opinions of authors expressed herein do not necessarily state or reflect those of the United States Government or any agency thereof. 


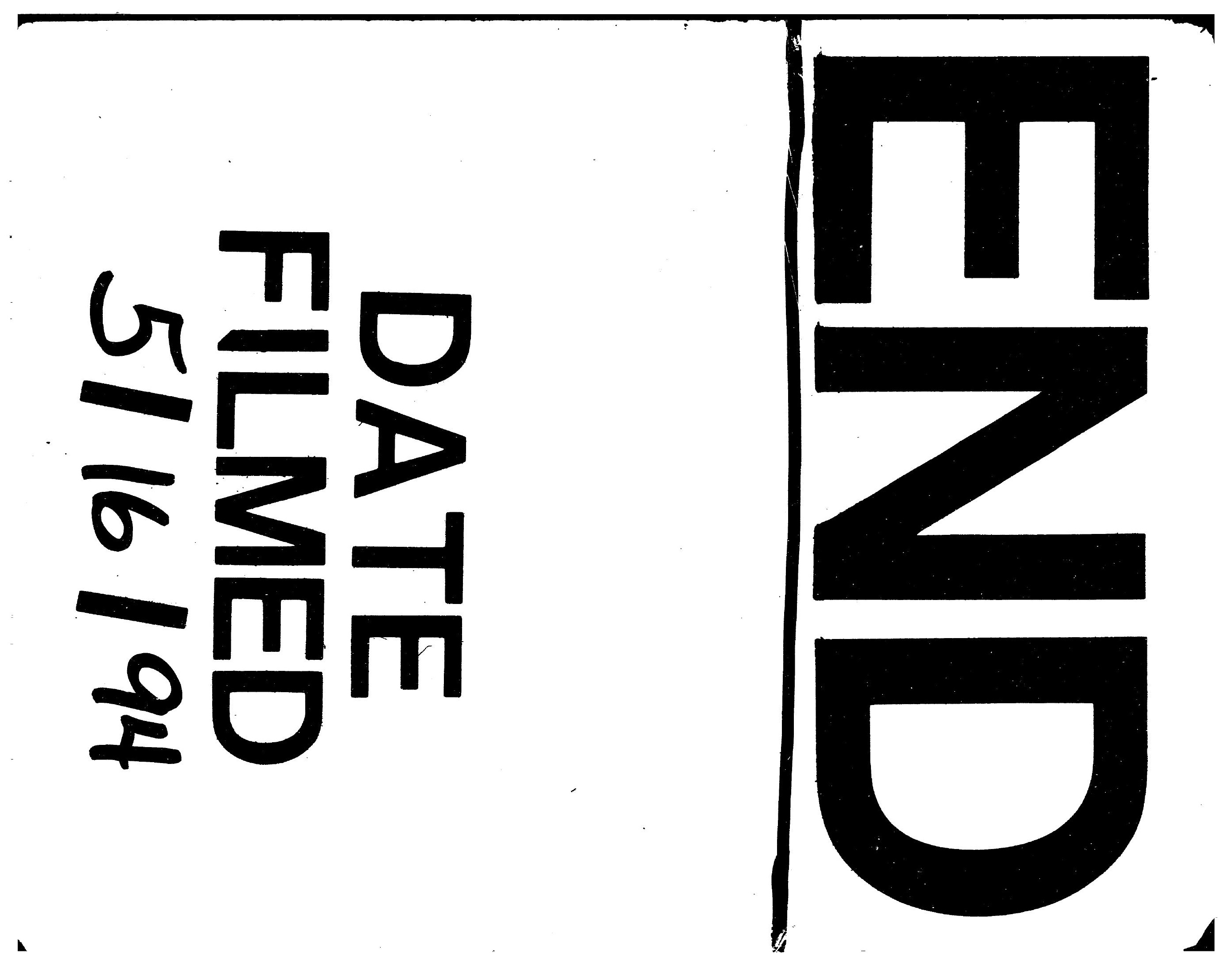




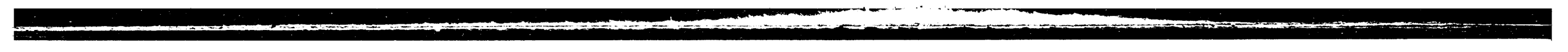

